\title{
Apego al cumplimiento de los indicadores de calidad en endoscopia del tracto gastrointestinal superior en el Hospital General Dr. Manuel Gea González
}

\author{
José R. Ramírez-Nava ${ }^{1 *}$, Roberto Délano-Alonso ${ }^{1}$, María del C. Ávila-Langarica², \\ Enrique Rentería-Palomo ${ }^{1}$, Ricardo Pineda-Leguízamo ${ }^{1}$, Carlos Valenzuela-Salazar ${ }^{1}$ y \\ José de J. Herrera-Esquivel ${ }^{1}$ \\ ${ }^{1}$ División de Cirugía Endoscópica; ²División de Endoscopia Gastrointestinal. Hospital General Dr. Manuel Gea González, Ciudad de México, México
}

\begin{abstract}
Resumen
La endoscopia del tracto superior digestivo se utiliza para el diagnóstico y tratamiento de trastornos digestivos. La calidad de la atención sanitaria puede medirse comparando el desempeño de un individuo o grupo con un punto de referencia determinado. Material y métodos: Se trata de un estudio descriptivo en el que se tuvo como referencia el registro de base de datos del Servicio de endoscopia del Hospital General Dr. Manuel Gea González. Resultados: Se revisaron 300 reportes de endoscopia, de los cuales 154 eran hombres (51.3\%) y 146 mujeres (48.7\%), con una mediana edad 56 años; de los 21 indicadores de endoscopia del tracto gastrointestinal superior que se pudieron evaluar, se cumplen 12 (57.1\%); La Sociedad Americana de Endoscopia Gastrointestinal y el Colegio Americano de Gastroenterología publicaron la primera versión de indicadores de calidad para endoscopia superior, actualizada con nuevos datos pertinentes en 2015, en los cuales se basaron los resultados. Conclusiones: Consideramos que el haber realizado este estudio es un parteaguas para conocer nuestras deficiencias en cuanto a la calidad de nuestros procedimientos, evaluar las razones del no cumplimiento de los indicadores y de esta manera implementar acciones de mejora, que lleven al cumplimiento sugerido por las guías.
\end{abstract}

Palabras clave: Indicadores. Calidad. Endoscopia gastrointestinal superior.

\section{Attachment to quality indicators in upper endoscopy at the Dr. Manuel Gea González General Hospital}

\begin{abstract}
The endoscopy of the upper digestive tract is used for the diagnosis and treatment of digestive disorders. The quality of health care can be measured by comparing the performance of an individual or group with a given benchmark. Material and methods: This is a descriptive study in which the database registry of the Endoscopy service of the Dr. Manuel Gea González General Hospital was taken as a reference. Results: 300 endoscopy reports were reviewed, of which 154 were men (51.3\%) and 146 women (48.7\%), with a median age of 56 years; of the 21 indicators of endoscopy of the upper gastrointestinal tract that could be evaluated, 12 (57.1\%) are met. The American Society of Gastrointestinal Endoscopy and the American College of Gastroenterology published the first version of quality indicators for higher endoscopy, being updated with new relevant data in 2015, on which the results were based. Conclusions: We consider that having
\end{abstract}

Correspondencia:

*José R. Ramírez-Nava

E-mail: jororana@gmail.com
Disponible en internet: 08-05-2020 Endoscopia. 2020;32(1):2-6 www.endoscopia-ameg.com
Fecha de recepción: 03-10-2019

Fecha de aceptación: 28-11-2019 DOI: 10.24875/END.19000029 pr Permanyer. Éste es un artículo open access bajo la licencia CC BY-NC-ND (http://creativecommons.org/licenses/by-nc-nd/4.0/). 
carried out this study is a watershed to know our deficiencies in terms of the quality of our procedures, evaluate the reasons for non-compliance with the indicators and thus implement improvement actions, which lead to the compliance suggested by the guidelines.

Key words: Indicators. Quality. Upper gastrointestinal endoscopy.

\section{Introducción}

La endoscopia del tracto superior digestivo se utiliza para el diagnóstico y tratamiento de trastornos del esófago, estómago e intestino delgado. Cuando se realiza de manera correcta, se considera un procedimiento seguro y tolerado en la evaluación del tracto gastrointestinal superior ${ }^{1,2}$.

En 2009 en EE.UU. se estimó que se realizaron 6.9 millones de endoscopias. Entre los años 2000 y 2010 este tipo de procedimiento se incrementó en un $50 \%{ }^{1}$.

La calidad de la atención sanitaria puede medirse comparando el desempeño de un individuo o grupo con un punto de referencia determinado, este punto de referencia o parámetro de comparación se denomina indicador de calidad. Los indicadores de calidad pueden reportarse como la proporción entre la incidencia del rendimiento correcto y la oportunidad de desempeño correcto, o como la proporción de intervenciones que logren un objetivo predefinido.

Los indicadores de calidad pueden dividirse en tres categorías:

1. Medidas estructurales. Evalúan las características del ambiente del sistema de salud.

2. Medidas de proceso. Evalúan el desempeño de la atención.

3. Medidas de resultado. Evalúan la atención que se ofreció.

El objetivo de nuestro estudio es conocer el apego al cumplimiento de los indicadores de calidad en las endoscopias del tracto gastrointestinal superior tras un programa de mejora en el Servicio de endoscopia del Hospital General Dr. Manuel Gea González.

\section{Material y métodos}

Se realizó el estudio descriptivo en el que se tuvo como referencia el registro de base de datos del Servicio de endoscopia del Hospital General Dr. Manuel Gea González, teniendo como población de estudio los reportes de endoscopia completos del tracto gastrointestinal superior durante el periodo del 1 enero al 30 de marzo del 2018, seleccionando un total de 300 reportes de endoscopias completos para análisis, siendo esta cantidad muestra por conveniencia.
Previamente al inicio de la investigación, se revisaron los indicadores de calidad de endoscopia de tracto gastrointestinal superior, de acuerdo con las guías de la Asociación Americana de Endoscopia Gastrointestinal ${ }^{1}$, haciendo énfasis en su aplicación en nuestro servicio.

Los ítems evaluados fueron los que se mencionan en la tabla 1, y del lado derecho se observa el porcentaje mínimo de apego sugerido.

Toda la información se obtuvo de los 300 reportes de endoscopia realizados, donde todos los datos requeridos estuvieron registrados en una base de datos y completos para realizar el análisis de los diferentes indicadores de calidad; en caso de no encontrar la información completa se excluía dicho estudio.

La base de datos del servicio se realiza semanalmente, introduciendo los datos de cada paciente y el resultado del estudio, junto con los indicadores de calidad a tomar en cuenta para endoscopia del tracto gastrointestinal superior.

Para el análisis se utilizó estadística descriptiva: proporciones o porcentajes según la escala de la variable.

\section{Resultados}

Se obtuvo una muestra por conveniencia, donde se revisaron 300 reportes de endoscopia en el periodo del 1 enero al 30 de marzo del 2018, de los cuales 154 eran hombres (51.3\%) y 146 mujeres (48.7\%), con una mediana de edad de 56 años (15-95) (Tabla 2). No se realizó exclusión de ningún reporte endoscópico debido a que se contaba con toda la información necesaria para el análisis en este trabajo.

De los 21 indicadores de endoscopia del tracto gastrointestinal superior que se pudieron evaluar, se cumplen 12 (57.1\%), en algunos de ellos se alcanza un 50\% del rendimiento sugerido por las guías.

En la figura 1 se desglosan cada uno de los indicadores evaluados. En el periodo preprocedimiento observamos que se logra la meta en 5 de 6 (83.3\%) indicadores de calidad, siendo el indicador número tres, el único que no logra la meta establecida.

En la figura 2 se muestran los resultados intraprocedimiento. En este periodo se excluye el indicador 17, ya que no es parte de los procedimientos realizados 
Tabla 1. Indicadores de calidad de acuerdo con las guías de la Sociedad Americana de Endoscopia

Periodo Indicador de calidad

Preprocedimiento

Indicaciones apropiadas y la indicación se documenta

Consentimiento informado

Antibiótico profiláctico

Antibiótico en pacientes cirróticos con hemorragia

Uso de IBP en pacientes con sospecha de sangrado por úlcera péptica

Inicio de fármacos vasoactivos en sospecha de sangrado de origen variceal

Intraprocedimiento

Frecuencia con la que se realiza un examen completo de esófago, estómago, duodeno, y se documenta

Entre las personas con úlcera gástrica sin hemorragia, frecuencia con la que se toman biopsias para excluir malignidad

Frecuencia con la que el esófago de Barrett se mide adecuadamente, cuando está presente

Sospecha de esófago de Barrett, se tomaron biopsias de dicho sitio

Frecuencia con la que se describe el tipo de sangrado gastrointestinal, su lesión, y se documenta la localización

Frecuencia con la que durante el estudio se observan úlceras pépticas, con al menos uno de los siguientes estigmas: hemorragia activa, vaso visible sin hemorragia (protuberancia pigmentada), coágulo adherido, mancha plana y base de fibrina limpia

Sospecha de esófago de Barrett, se tomaron biopsias de dicho sitio

$>98 \%$

Frecuencia con la que se describe el tipo de sangrado gastrointestinal, su lesión, y se documenta la localización

Frecuencia con la que una segunda modalidad de tratamiento se utiliza cuando se usa la inyección de epinefrina

Frecuencia con la que se utiliza la ligadura de varices como primera modalidad de tratamiento para el tratamiento endoscópico de varices esofágicas

$>80 \%$

$>98 \%$

$>98 \%$

$>98 \%$

$>98 \%$

$>98 \%$

$>98 \%$

$>80 \%$

$>98 \%$

$>80 \%$

$>98 \%$

$>98 \%$

$>80 \%$

$>98 \%$

$>98 \%$
Objetivo

Frecuencia con la que se recomienda la terapia con IBP para pacientes que se sometieron a dilatación por estenosis esofágica péptica

Frecuencia con la que los pacientes diagnosticados con úlcera gástrica o duodenal son instruidos $>98 \%$ para tomar IBP

Frecuencia con la que se planea evaluar infección por Helicobacter Pylori en pacientes con diagnóstico de úlcera gástrica o duodenal

Frecuencia con la que los pacientes con hemorragia recurrente secundaria a úlcera péptica después de tratamiento endoscópico se someten a una nueva endoscopia

Frecuencia con la que los pacientes son contactados para documentar la aparición de eventos

\section{$>98 \%$}

$>98 \%$

NA

IBP: inhibidor de la bomba de protones; NA: no aplica.

durante este estudio; observamos que en solo cuatro de los indicadores se logran las metas establecidas, siendo el $40 \%$ de los indicadores en este periodo, el otro $60 \%$ con resultados menores a las metas establecidas, siendo el indicador 15 en donde obtenemos el menor porcentaje, con un $33.3 \%$.

En la figura 3 se muestran los resultados posprocedimiento. En este periodo encontramos que se cumplen las metas en el $60 \%$ de los indicadores, obteniendo el menor de los porcentajes (con un 2.3\%) el indicador 22, sin embargo, no existe aún una meta establecida por la Sociedad Americana de Endoscopia Gastrointestinal (ASGE) para tomar en cuenta el resultado; por otro lado nos indica un pobre seguimiento de los pacientes sometidos a endoscopia gastrointestinal alta. 
Tabla 2. Resultados de los indicadores de calidad en el Hospital General Dr. Manuel Gea González

\begin{tabular}{|c|c|c|}
\hline \multicolumn{3}{|c|}{ Indicadores de calidad (enumerados según guías ASGE) } \\
\hline Preprocedimiento & Intraprocedimiento & Posprocedimiento \\
\hline 1. $99.7 \%(>80 \%)$ & 7. $97.7 \%$ (> 98\%) & 18. $100 \%$ (> 98\%) \\
\hline 2. $100 \%(>98 \%)$ & 8. $55.6 \%$ (> $80 \%)$ & 19. $100 \%$ (> $98 \%)$ \\
\hline 3. $86.7 \%$ (> 98\%) & 9. $80.0 \%$ (> 98\%) & 20. $60.6 \%(>98 \%)$ \\
\hline 4. $100 \%$ (> 98\%) & 10. $80.0 \%(>90 \%)$ & 21. $100 \%$ (> 98\%) \\
\hline 5. $100 \%$ (> 98\%) & 11. $98.0 \%(>80 \%)$ & 22. $2.3 \% \mathrm{~N} / \mathrm{A}$ \\
\hline \multirow[t]{6}{*}{ 6. $100 \%(>98 \%)$} & 12. $100 . \%(>98 \%)$ & \\
\hline & 13. $100 \%$ (> 98\%) & \\
\hline & 14. $100 \%(>98 \%)$ & \\
\hline & 15. $33.3 \%$ (> 98\%) & \\
\hline & 16. $71.4 \%$ (> 98\%) & \\
\hline & 17. NA (> 90\%) & \\
\hline
\end{tabular}

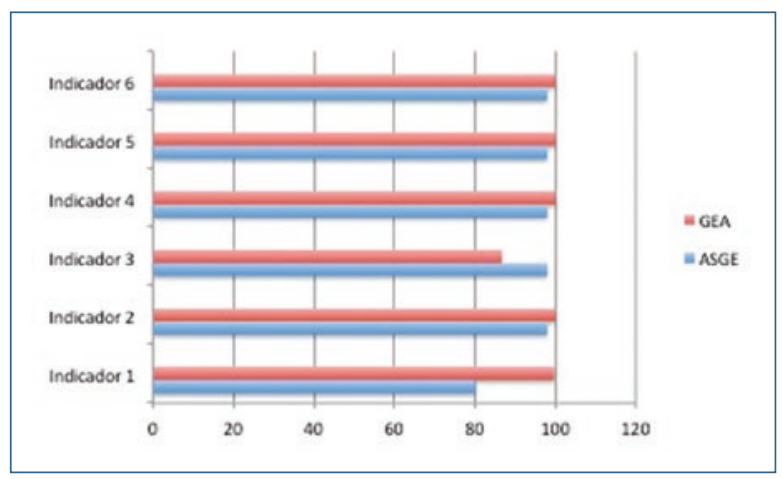

Figura 1. Resultados preprocedimiento. GEA: Hospital General Dr. Manuel Gea González; ASGE: Sociedad Americana de Endoscopia Gastrointestinal.

\section{Discusión}

En 2006, la ASGE y el Colegio Americano de Gastroenterología (ACG) publicaron la primera versión de indicadores de calidad para endoscopia superior, actualizada con nuevos datos pertinentes en 2015.

Cada indicador de calidad se clasificó como un resultado o una medida del proceso.

Aunque se prefieren los indicadores de calidad de los resultados, algunos pueden ser difíciles de tomar en la práctica clínica habitual, ya que se necesita un análisis de grandes cantidades de datos y el seguimiento a largo plazo puede ser confundido por otros factores. De tales casos, el grupo de trabajo consideró

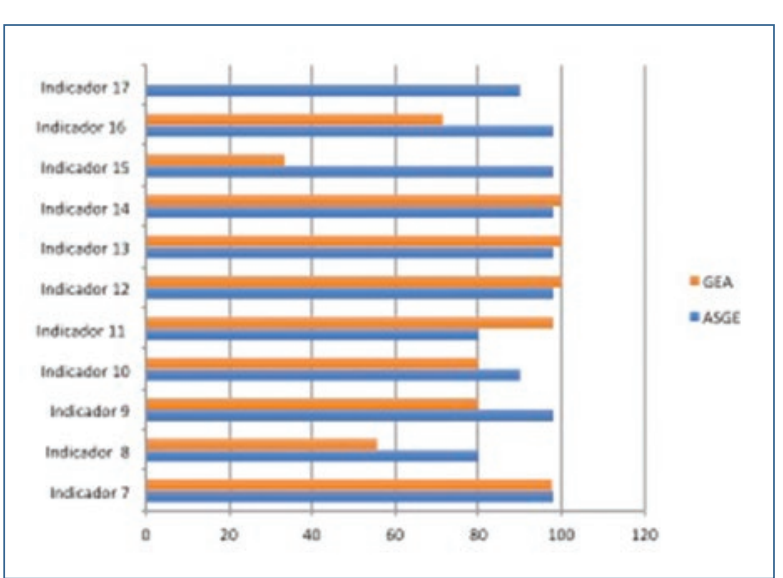

Figura 2. Resultados intraprocedimiento. GEA: Hospital General Dr. Manuel Gea González; ASGE: Sociedad Americana de Endoscopia Gastrointestinal.

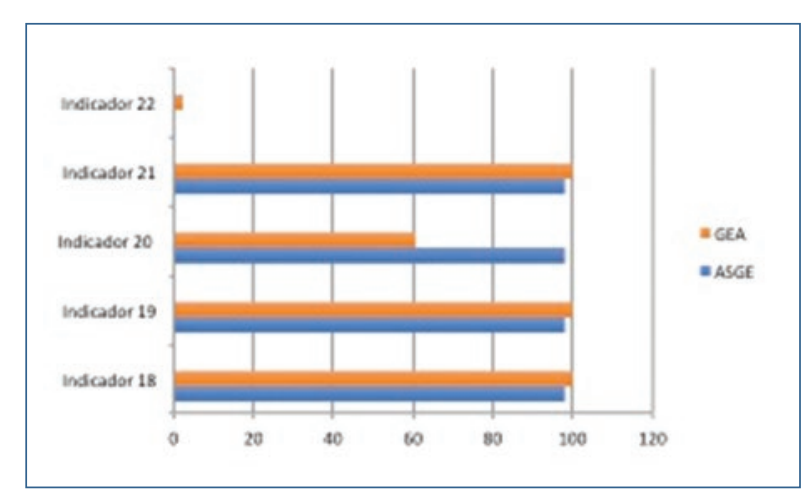

Figura 3. Resultados posprocedimiento. GEA: Hospital General Dr. Manuel Gea González; ASGE: Sociedad Americana de Endoscopia Gastrointestinal.

razonable utilizar indicadores de proceso, como medidas sustitutivas en la endoscopia de alta calidad'. En las guías de la Sociedad Americana de Endoscopia Gastrointestinal, los indicadores de calidad se dividieron en 3 periodos de tiempo: preprocedimiento, intraprocedimiento y posprocedimiento (Tabla 1).

De acuerdo con nuestros resultados, de los 21 indicadores de endoscopia del tracto gastrointestinal superior que se pudieron evaluar, se cumplen 12 (57.1\%); en algunos de ellos se alcanza un $50 \%$ del rendimiento sugerido por las guías.

En 2009, en el Hospital de Especialidades del Centro Médico Nacional la Raza del IMSS, se llevó a cabo un estudio acerca del registro de indicadores de calidad en esofagogastroduodenoscopia. El estudio tomó en cuenta solo los siguientes indicadores: consentimiento informado, examen completo, medición de la extensión 
de esófago de Barrett, toma de biopsias en esófago de Barrett, toma de biopsias en úlcera gástrica, descripción y localización de lesiones en hemorragia de tubo digestivo alto, tratamiento endoscópico para hemorragia de tubo digestivo alto, documentación de hemostasia en pacientes en quienes se aplicó tratamiento hemostático, recomendación de inhibidor de la bomba de protones en pacientes a quienes se les realizó dilatación esofágica por estenosis péptica y en pacientes con úlcera péptica. De los 10 indicadores de calidad evaluados, nueve tuvieron un porcentaje de registro por arriba del $80 \%$ y seis por arriba de $90 \%$. Solo uno de ellos estuvo por debajo del $30 \%$ y correspondió a la recomendación del inhibidor de la bomba de protones en pacientes con diagnóstico de úlcera péptica.

Con base en los resultados, sus autores concluyeron que la frecuencia en el registro de los indicadores de calidad evaluados es alta para indicadores relativos a la hemorragia de tubo digestivo alto, el examen completo y en esófago de Barrett. Las recomendaciones de tratamiento farmacológico en los trastornos de origen péptico es un tópico controvertido que debe mejorarse. No se puede establecer si no se cumplió con todos los indicadores, ya que cabe la posibilidad de que se hubieran realizado pero no registrado en los informes. Sin embargo, una atención de calidad incluye el registro adecuado de todas las acciones ${ }^{3}$.

Recientemente, en 2017, Córdova, et al. publicaron un estudio comparativo de indicadores de calidad en la esofagogastroduodenoscopia tras un programa de mejora en un hospital terciario, en el cual se reportó que tras el programa de formación, se observó una mejoría significativa en los siguientes indicadores: indicación documentada (93 vs. 99.8\%), exploraciones completas documentadas (94.7 vs. $97.3 \%$ ), actuación correcta (63.7 vs. $87.9 \%$ ), toma adecuada de biopsias según protocolos ( 57.9 vs. $83.8 \%$ ), fotodocumentación de lesiones descritas (84.1 vs. $94.9 \%$ ), fotodocumentación por segmentos (52.9 vs. $70.5 \%$ ) y valoración global correcta ( 56.9 vs. $90.5 \%$ ). La toma de biopsias para enfermedad celiaca, la indicación documentada, la exploración completa y la actuación correcta, si procedía, consiguieron superar el estándar recomendado. Concluyen que tras un programa de formación muy sencillo, mejoran los indicadores de calidad de la esofagogastroduodenoscopia, alcanzando la mayoría los estándares recomendados por la ASGE y el $\mathrm{ACG}^{4}$.

\section{Conclusiones}

Según los resultados obtenidos y observados, se cumplen únicamente 12 de 22 de los indicadores de calidad establecidos por la ASGE (57.1\%), recalcando que el periodo intraprocedimiento es el que menor número de metas cumplidas obtuvo, por lo que es necesario puntualizar las deficiencias ocurridas, generar un mejor plan y lograr mejor calidad en nuestros procedimientos.

Consideramos que el haber realizado este estudio es un parteaguas para conocer nuestras deficiencias en cuanto a la calidad de nuestros procedimientos, evaluar las razones del no cumplimiento de los indicadores y de esta manera poder implementar acciones de mejora, que lleven al cumplimiento sugerido por las guías, para poder ofrecer un servicio de calidad y calidez a nuestros pacientes.

\section{Responsabilidades éticas}

Protección de personas y animales Los autores declaran que para esta investigación no se han realizado experimentos en seres humanos ni en animales.

Confidencialidad de los datos Los autores declaran que han seguido los protocolos de su centro de trabajo sobre la publicación de datos de pacientes.

Derecho a la privacidad y consentimiento informado Los autores declaran que en este artículo no aparecen datos de pacientes.

\section{Bibliografía}

1. Rizk M, Sawhney M, Cohen J, Pike I, Adler D, Dominitz J, et al. Quality indicators for GI endoscopic procedures. Gastrointes Endosc. 2015;81:17-30.

2. Bretthauer M. Requirements and standards facilitating quality improvement for reporting systems in gastrointestinal endoscopy: European Society of Gastrointestinal Endoscopy (ESGE) Position Statement. Endoscopy. 2016;48:291-4.

3. Trujillo-Benavides OE, Navarro-García AM, Baltazar-Montúfar P. Registro de indicadores de calidad de la esófagogastroduodenoscopia en el Hospital de Especialidades del Centro Médico Nacional La Raza del IMSS. Gastroenterol Mex. 2009;74:302-5.

4. Córdova H, Sánchez-Montes C, Delgado-Guillena PG, Morales VJ, Sendino O, González-Suárez B, et al. Indicadores de calidad en la esofagogastroduodenoscopia: estudio comparativo de los resultados tras un programa de mejora en un hospital terciario. Gastroenterol Hepatol. 2017;40:587-94. 